\title{
Uterine Corpus Carcinoma and Carcinosarcoma pN0 TNM Finding v8
}

National Cancer Institute

\section{Source}

National Cancer Institute. Uterine Corpus Carcinoma and Carcinosarcoma pNO TNM

Finding v8. NCI Thesaurus. Code C139778.

Uterine corpus carcinoma or carcinosarcoma with no regional lymph node metastasis.

(from AJCC 8th Ed.) 